\title{
II.
}

Ueber den Strontian-Gehalt des slragonit, nach den Verfuchen der Herren Bucholz und Meifsner,

von

\section{GAY - LUSSAC *).}

Herr Stromeyer hatte aus feinen Zerlegungen vieler Arragonite den Schluls gezogen, dalis alle Arten von Arragonit 1,2 oder 4 Procent kohlenfauren Strontian enthalten, und dal's, fo gering diele Menge auch fey, fie fich doch nicht für blos zufällig vehmen lalle, fund höcht wahrfcheinlich die Verfchiedenheit zwilchen den phyfikalifehen Eigenfchaften des Arragonit und des rhomboidalen Kalkfpaths begründe. Herr Stromeyer bat zwar in der fogenanuten Eifenblüthe, einer Stalactilart, welche die Herren Bouruon und Cordier zu dem Alragonite rechnen, noch in dem Mineral der Porta Weftphalica bei Minden, welche Andere fuir Arragonit ausgegeben hatten, Strontian finden kömuen; bei

") Frei bearicitet nacb den Annales de Chinie et de Dhy/gque 1816. t. 2., von

Gilbert. 
genauerer Uuterfuchung diefer beiden Minerale überzeugte er lich aber, 'dal's lie eine rhomboidilche Structur liaben, daher er es auch aufser Zureifel hält, dafs fie zu dem Kalklpalhe und nicht zu den Arragonite gehören.

Enthielte aller Arragonit Strontian, fo wäre es ganz natürlich, hierin die Urlach der phyfikalifchen Eigenl'chaften zu letzen, welche ihn von dem rhomboidalen KaIklpathe unterfcheiden. Denu in einem zulummengefetzten Körper müfen alle Beftandheile defielben $z u$ Ceinen Eigenlchaften beitragen, und wir fehen, dafs fehr geringe Mengen $\mathrm{K}$ ohlenltoff, die nicht minder beträchtliche Verfchiedenheit wifchen Eifen und Stahl hervorbringen. Man darf überhaupt nicht glauben; dafs ein Körper, der in einer Verbindung nur in einer fehr kleinen Menge, und, wie es licheint, nach keinem beftimmlen Verhültnille, vorhanden ilt; blos zufillig und ohne Einflufs auf die Eigenfchaften der Verbindung fey. Nach der Idee, welche wir uns von den Kürpern machen, können fich ihre Molecüle in Gruppen vereinigen, und diefe Gruppen aufs neue als kleinfte Theilchen fich mit fremdartigen Molecülen verbinden. Diefe Verbindungsart finden wir in Körpern, welche aus einer grofsen Menge von Beftandtheilen zufammengeletzt lind, und jch halle es für wahrfcheinlich, dals fich die Molecüle in Gruppen vereinigen, die lich bei den Verbindungen, fey es zu folge ihrer Geftalt, oder der Kräfte, die fie belceben, wie einfache Molecüle ver-

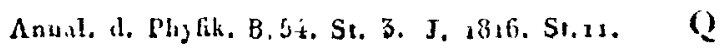




\section{$\left[\begin{array}{lll}234 & 1\end{array}\right.$}

halten. Auf diele Art wenigftens wärde ich mir die Verbindung von Eilen und Kohlenftoff im Stahle, und die des kohlenfauren Kalks uad kohlenfauren Strontians im Arragonit denken; und ich zweille nicht, dafs die Gefetze der befirmmten Proportionen nicht auch hicr fich wirkfum zeigen.

Es giebt aber noch cine andere Art, die Verbindungen zweicr Körper in to verlchiedenem Verhallnille, zu betrachten, auf die man bis jetzt nicht aufinerkfam gewefen ift. Bringt mankryfalle von Alaun mit Ammoniak- Bafis in eine gefältigte Auflöfuug von Alaun mit Kali- Balis, fo wachlen fie in ihr regelmälsig fort durch parallele Lagen; und bringt man fie dann in eine gefistligte Aufiolun erfien Art von Alaun, fo wachfen fie auch in diefer wieder regelnälsig fort, fo dals man fehr regelmäfsige Krylialle crhält, welche aus Lagen von verfchiedener Natur zufammengeletzt find. Giefst man beide Auflöfungen zufammen, fo wachfen auch dann noch in ihr die Kryftalle, indem lie fich der Alaun - Molecüle mit Kali - Bafis und derer mit Ammoniak - Bafis ohue Auswahl bemüchtigen, und fich dann allo nach fchr variablen Verhäluillen gemifcht zeigen werden. Nieler Litolg hat leinen Grund olfenbar darin, dafs die Molecüle beider Arten von Alaun einerlei Geftult, und anftreitig auch einerlei Kräfte befitzen, und dals es daher fiir das Anwachlen des Kryftalls gleiclsgültig ilt, ob er lich eines Moleciils der einer oder der andern Alaunart temächligt. Su oft alfo diefer limltand flatt findet, 


\section{[ 235 ]}

das heifst, wenn Molecüle verfchiedener Natur auf einerlei Weife zur Bildung eines Kryftalls beitragen können, fo mufs man erwarten, zufimmengelelzte Körper nach jedem Verhältuille entltehen zu fehen. Dieles ilt alfo eine den fohon bekannten Urfachen noch beizufigende Urfach von Abweichungen von dem allgemeinen Gefelze der beftimmten Proportiouen.

Was den Arragonit betriflt, fo laffen fich jedoch dia eigenthümlichen Eigenlichaften dellelben nicht mil Hrn. S tro me yer daraus erklüren, dafs ihm Strontian beigemifcht ift. Dewn es, ift nicht in allen Arten von Arragonit Strontian enthalten, wie die Verfuche lehren der HH. Bucholz in Erfurt und Meil'sner [aus Halle, damals Herra Bucholzen's Gehülfe, jetzt wieder in Halle.] Die Abhandlung, in der lie diefe ihre Verfuche bekannt gemacht haben (im Schweigger'fchen Jaurn. für Chemie $\Gamma h, 13$.$) nimmt 71$ Seiten ein, und lind in einem folchen Delail befchrieben, dafs fie das gröfste Vertrauen einflölien wïrden, wenn auch nicht der Name Bu cholz ihnen zur Empfehlung diente. Ich will die Refultate derlelben hicr lo kurz als möglich angeben:

Gleich, als HerruStrom yer's Entdeckung bokannt wurde, hatle fich Hert Bucholz nmfonft beinüh, in einigen Arteu von Arragonit Strontian aufzutinden, fowohl durch das Stromeyer'fche Verfahren, als durch :Auflöfen des Arragouit in Salpeterfiure, 'Lerflörung des entftandeQ2 


\section{[ 236 ]}

nen fal peterfauren Salzes durch Erhitzen, und durch Autlöfen des Strontian in fo wenig Wafter, dals der Kalk nicht aufgelöft wurde. Da Heır Bucholz fürchtete, nicht unter denfelben Umliänden als Hr. Stromeyer gearbcitet zu haben, fo nahm er Herin Meil'sner zu Hülfe, um eine grolse Menge von Arragoniten zu analyfiren.

Es kam ihnen zuerli darauf an, die beiden eben erwähnten Arten der Avalyfe zu prïfen, und dabei bedienten fie fich des Arragonits vou Neumarkt, weil fie davon eine bedeutende Menge belarsen. Sie lölten ihn in Salpelerlüure auf. Das Salz, welches entliand, war vollkommen neutral. Der Rückftand der bis zur 'Trocknil's abgedampften Auflüfung wurde zu einem feinen Pulver zerrieben, und in einer glälernen Flafche mit dem Dreifachen leines Gewichts abloluten Altohol übergofien, welches das, fchicklichlte Verhältnifs ift, um die MalTe aufzulöfen. Es löfie fich alles auf unter Erhitzung, bis auf einen kaun wahrnembaren Rückftand; es lobied lich aber weder durch Ruhe, noch durch müglichit langlames Abdampfen falpeterfaurer Strontian daraus ab. Bei fortgefetztem Abdamp a erhielten lio eine kryliallinilche Malle falpeterfauren Kalks, welche auf Filtrirpapier der Luft ausgefelzt zerfiofs, ohne den geringlien Riuckfiand zu Jallen. Sie verfuchten nun das Buchalz'lche Verfahren, mittellt deflen Herr Gelle n ein wenig Strontian in dicfer Arragonit - Art gefunden haben wolle, konn- 


\section{$\left[\begin{array}{lll} & 237 & \end{array}\right]$}

ten aber bei aller Aufmerkfamkeit in zwei $V$ erfuchen nichts davon entdecken.

Sie finden, dals abfuluter Alkohol in der gewöln nlichen Temperatur ren Strontian auflölit, und dafs die Anwefenheit von la! pelcrlaurem Kalk die Auflöslichkeit deflelben eher vermindert als vermehrt. Dagegen lïft fich diefes Jetzlere Salz in Cehr grofser Menge in abfolutem Alkohol auf. Mit $\frac{\pi}{2}$ Theilen Alkohol ilt dic Aullïfung Syruparlig und geht fobwer durch das Filtrum; mit 2'Theilen lïlst tie lich lehr leicht filtrirer.

Da der Strontian im Arragonit an Kohlcraïnre gebunden if, bei dem Stromeyer'fchen Verfaliren aber die Menge deffelbeu nach der Menge lulpeterfauren Strontians, welche man erhäl, beliinmt wird, lo verwandelten die HH. Bucholz und IJ cilsner kryltallifirten falpeterfauren Strontian in kollenfauren, und dabei fauden lie, dal's !oo Theite falpelerfurer Strontian 69 'Theile rollhommen trochuen kohlenfuren Strontian geben.

Nach dielen vorliuligen Verfuchen wen. delen fich nun beide Chemiker zu der Analyle von 12 verfchiedenen Arragonit-Arten, welche lie befalsen.

Der Arragonit von Neumarkt gab bei einer reuen Analy le wicderum keine Spur von Strontian. Er belteht ganz aus kolilenfaurem Kalk, dem eiue fehr geringe Menge Gyps beigenengt if.

Der Arragonit aus Spanien gab kohlenlauren 


\section{[ 238 ]}

Strontian, die Menge deffelhen ift aber in verfchicdenen Stücken verl'chiuden. Keiue Art vou Arrugonit ilt ganz reiu. In denen, welche keinen kohleuluuren Strontian euthalten, crletzt etwas Gyps die Stelle defrelben, manchmal auch etwas Eifell und 'Thonerde, wie in dell Arragonit rou Limburg:

Das Refullat diefer Unterfuchungen der HH. Bucholz und Meifsuer ift folgendes: Jinige Arragovite enthalien kuhloufauren Strontian, andere nicht; und doch felieinen dio letztern allo refintlichen mineralogiliben Charaktere, welche cien erflern eigen liud, zu buliten. Der Strontian ift daber für cine blos zulällige Beimengung zu hallen, velche aa der Kryflallifation des Arragonit keiuen Autheil hat. Auch ift die Menge deffelben immer nur lehr klein und fehr variabel, und oft fehlt er ganz. Keinen Strontian enthalten dio Arragonite von Neumnrkt, Salfeld, Minden, La/tonne und Limburg. Die andera fieben Arragonite enthalten folgende Mungen ron Stroution in 100 'Theileu: Arragonit aus Spanien $\$$ bis $\frac{3}{4}$ 'Theile, und eine audere Art von daher in fehr fchönen Kryllallen 'Theil; Arragonit aus

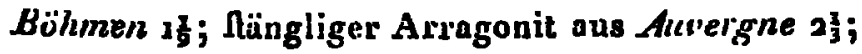
ein auderer fronsijffcher Arragonit, unbekaunt woher, ${ }_{\frac{x}{3}}^{\frac{x}{3}}$ Theile; HrahligerArragouit von Büdheim $2 \%$ Theile.

Ditlo Refultate, welcho ron denen des Herrn Stromeyer lelur perfichicden find, beweilen, dal's 


\section{[ 239 ]}

der kohlenfaure Strontian nach keinen foften und beltimmten Verhältnillen in den Arragoniten, die ihn enthalten, fondern nur zufällig rorkömmt.

G. $\boldsymbol{L}$.

\section{III.}

Bemerhungen über den vorhergehenden $A: \iint j a / z$, und Rechtfertigung feiner Meznung von der

Natur des Arragonits,

$$
\text { von dem }
$$

Prof. Strourkyer in Göttingen.

(In einem Briefe an den Profeffur Gilbert.)

Gïtling, en ilen 29. Sept. 1816 .

Sie fordern mich auf, Ihuen meine Gedauken über deu Ihrem Briefe in der Uoberfelzmng beigelegien Auifutz zu fugen, worin flerr $G$ a $y-L u \int C a c$, in den Annales de Chimie, die Verliche der Herren Bucholz und M eil'sner über den Arragonit in einem kurzen Auszuge millhuilt. Zwar hảbe ich lichon in demfelben Bande des Schweigger'fchen Journals, worin die Analyle des Arragunits der 1H. Bncholz und Meifsuer enthallen ifi, (B. 12. 